\title{
Emission-intensity-enhanced GaN-based LED based on multilayer grating structures
}

\author{
Xin $\mathrm{Li}^{1}$, Dejie $\mathrm{Sun}^{1}, \mathrm{Kun} \mathrm{Han}^{1}$, Lijun $\mathrm{CaO}^{1}$, Shiliang GuO ${ }^{2}$, Zhiquan $\mathrm{Li}^{2}$ \\ ${ }^{1}$ School of Mathematics and Information Science and Technology, \\ Hebei Normal University of Science and Technology, \\ Qinhuangdao 066004, China \\ ${ }^{2}$ Key Laboratory of Measurement Technology \& Instrumentation of Hebei Province, \\ Yanshan University, Qinhuangdao 066004, China
}

\begin{abstract}
A novel surface-plasmon-enhanced GaN-LED is proposed to improve the emission efficiency of the traditional LED. The $\mathrm{SiO}_{2}$ film, Ag triangular structure and ITO film were coated on the rectangularly-patterned $\mathrm{p}-\mathrm{GaN}$ layer sequentially, which can form the quasi-symmetrical waveguide structure to enhance the internal quantum efficiency and the light extraction efficiency. The COMSOL software is used to simulate the LED structure. The radiated powers, absorbed powers and distribution of electric field are obtained and analyzed. The results reveal that emission efficiency of the proposed GaN-LED can be greatly improved.
\end{abstract}

Keywords: light-emitting diode, surface plasmons, grating, quantum wells.

\section{Introduction}

GaN based light-emitting diodes (LEDs) are widely used in lighting, communication and other fields because of their advantages such as small size, high efficiency and high reliability [1- 1 ]. But for now, the application of LED in home lighting is far less popular than the traditional light source. This is mainly due to the large difference in refractive index between $\mathrm{GaN}$ and air. The total reflection of light at the interface causes most of the light to be reflected and cannot be effectively emitted, and finally converted into thermal energy. As a result, only a small fraction of the light can be converted into the usable optical power radiation. This makes the light extraction efficiency greatly reduced, and the LED is always in a high temperature working state, shortening its service life. In addition, Fresnel reflection can also cause some loss of light, resulting in a decrease in light extraction efficiency. Therefore, designing a LED structure, reducing the thermal energy loss inside the LED, and improving the light extraction efficiency will be an important breakthrough in solving energy utilization problems. The integration of surface plasmons (SPs) and LED provides a good approach to achieve LED de- 
vices with higher efficiency and higher modulation bandwidth. In 2004, Окамото et al. deposited three different metal layers (including $\mathrm{Ag}, \mathrm{Al}$ and $\mathrm{Au}$ ) on InGaN quantum wells (QWs), and systematically studied the SPs coupling enhanced luminescence. The enhancement effect of the metal layer on the emission efficiency of the LED is fully verified. Their results showed that the enhancement effects of different metals are different, wherein the Ag metal layer has the most obvious effect and can achieve a maximum enhancement effect of 14 times. Subsequently, the group studied the coupling distance between the metal layer and the active layer, and finally verified that the SPs coupling belongs to the near-field effect. The larger the distance between the metal layer and the active layer, the less obvious the near-field effect is [ [5]. In 2005, Окамото et al. redesigned the experiment to investigate the relationship between SPs coupling and the spontaneous emission rate of the internal excitons, and finally verified the enhancement effect of SPs on the spontaneous emission rate of the excitons in the LED [6]. In 2007, the Окамото team continued to expand the research object to metal gratings, and designed a sub-wavelength metal grating structure on the surface of the metal layer [7]. When a sub-wavelength metal grating is added, the efficiency of SPs conversion to light can be improved. In addition, the metal grating structure can control the SPs radiation, to exit in the direction of $21^{\circ}$. In 2007, YeH et al. studied the effect of ohmic contact on the enhancement of SPs [ $[$ ]. The results show that the presence of ohmic contact will consume a large portion of the SPs energy. Therefore, when designing the LED structure, it is necessary to add an insulating layer structure to eliminate the influence of ohmic contact, thereby obtaining better emission efficiency. Compared with the metal film structure, the advantages of metal particles are more obvious. After the metal particles are excited, a localized surface plasmon (LSP) is generated, which has a high scattering rate and an adjustable resonance frequency. In 2008, YEH et al. fabricated an Ag nanoparticle array structure on the surface of the $\mathrm{p}-\mathrm{GaN}$ layer by a special process, making full use of the coupling of LSP, so that the LED obtained a 120\% enhancement effect [9]. In 2010, in order to further reduce the distance between the metal particles and the active layer, CHO et al. embedded the metal particles into $\mathrm{p}-\mathrm{GaN}$ within $30 \mathrm{~nm}$ of the active layer, which greatly reduced the distance between the metal particles and the active layer, and ultimately increases the emission efficiency of the LED by $38 \%$ at normal current [10]. However, this method greatly reduces the quality of the $\mathrm{p}-\mathrm{GaN}$ material and affects the electrical characteristics of the LED device. In 2011, CHO et al. improved the above method to introduce the $\mathrm{SiO}_{2}$ nanodisk into the structure of the LED device, thus avoiding the influence on the LED characteristics and further increasing the emission efficiency of the LED by $72 \%$ [11]. In 2010, SHEN et al. studied the effects of the $\mathrm{SiO}_{2}$ on polarized output of a LED with SP coupling, and proved that the intermediate $\mathrm{SiO}_{2}$ layer can effectively improve the emission efficiency of LED [12]. In 2010, KAO et al. proposed a SP-enhanced GaN -based LED with Ag nanotriangle array by nanosphere lithography, which can provide a reliable method for making Ag nanotriangle structure [13]. In 2013, ZHANG et al. proposed a SP-enhanced GaN-LED based on the quasi-symmetrical planar waveguide structure and a SP-enhanced GaN-LED based on a multilayered M-shaped nano-grating, 
and the light extraction efficiency in those structures are effectively improved $[\underline{14}, \underline{15}]$. In 2014, ZHU et al. proposed a SP-enhanced GaN-LED based on the multilayered rectangular nano-grating, which can further enhance the emission efficiency of the LED [16]. In 2018, YAO et al. fabricated a metal grating on the top of the LED, using a certain grating period to compensate for its momentum mismatch, greatly enhancing the coupling effect between SPPs and QWs, thereby improving the luminous performance of the LED [17]. In 2020, HoNG et al. used the colloidal Ag nanoparticles to carry out the aerodynamic spraying experiments, which proved the dependence of LSPs-enhanced near-ultraviolet LED on the thickness of $\mathrm{p}-\mathrm{GaN}$ spacer. After the introduction of the colloidal Ag nanoparticles, 10 and $20 \mathrm{~nm}$ thick $\mathrm{p}-\mathrm{GaN}$ spacers can increase the internal quantum efficiency and reduce its effective exciton lifetime, and its internal quantum efficiency increased by $18.8 \%$ and $24.2 \%$, respectively [18].

In this paper, we demonstrate a novel surface-plasmon-enhanced GaN-LED. Our structure contains a $\mathrm{SiO}_{2}$ film of low refractive, an Ag layer and an ITO layer of high refractive coated on the rectangularly-patterned p-GaN layer sequentially. The Ag layer is used to induce SPs and enhance the internal quantum efficiency. The $\mathrm{SiO}_{2}$ film can suppress the absorption loss of SPs, and further improve the light extraction efficiency and the SPs extraction efficiency. The ITO layer can be used as a buffer layer of LED for its high light transmittance and conductivity. In addition, the ITO layer and the $\mathrm{SiO}_{2}$ film on both sides of the Ag layer can form a quasi-symmetric waveguide structure, which can extend the near-field distribution range of the local electric field, reduce the loss and absorption of the metal to the SPs, and improve the extraction efficiency of the SPs.

\section{Fabrication}

The comparison structure and the novel structure are shown in Figs. $1 \mathbf{a}$ and $1 \mathbf{b}$, respectively. Firstly, a 400-nm-thick n-GaN layer, an $\operatorname{InGaN}(3 \mathrm{~nm}) / \mathrm{GaN}(5 \mathrm{~nm})$ multilayer QWs (with a center wavelength of $460 \mathrm{~nm}$ ), and a 120 -nm-thick p-GaN layer
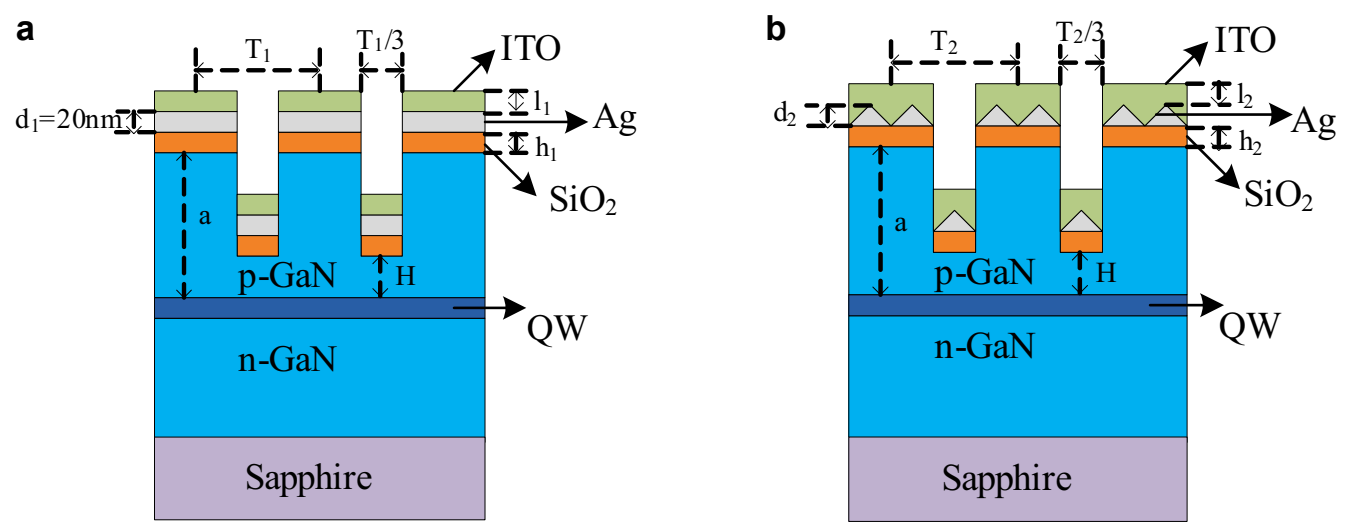

Fig. 1. The comparison structure (a), and the novel structure (b). 
$(a=120 \mathrm{~nm})$ are deposited on a sapphire substrate. To make the comparison with the novel structures, the surface of the $\mathrm{p}-\mathrm{GaN}$ is covered with an anode $\mathrm{Al}_{2} \mathrm{O}_{3}$ mask $(300 \mathrm{~nm})$, and the nano-grating structure is etched on the surface of the $\mathrm{p}-\mathrm{GaN}$ layer by inductively coupled plasma (ICP) etching, the etching depth of the nano-grating structure is $90 \mathrm{~nm}$, the distance between the grating ridge and the QW is $30 \mathrm{~nm}(H=30 \mathrm{~nm})$, and the duty ratio is $1 / 3$. For fabricating the comparison structure, a $\mathrm{SiO}_{2}$ film, an $\mathrm{Ag}$ film, and an ITO film are deposited on the nano-patterned p-GaN layer sequentially, as shown in Fig. 1a. The period of the nano-grating structure is $T_{1}$, the thicknesses of the $\mathrm{SiO}_{2}$ film, Ag film and ITO film are $h_{1}, d_{1}\left(d_{1}=20 \mathrm{~nm}\right)$, and $l_{1}$, respectively. For fabricating the novel structure, a $\mathrm{SiO}_{2}$ film, an $\mathrm{Ag}$ triangle structure and an ITO layer are deposited on the nano-patterned p-GaN layer sequentially, as shown in Fig. $1 \mathbf{b}$. The period of the nano-grating structure is $T_{2}$, the thickness of $\mathrm{SiO}_{2}$ film is $h_{2}$, the height of the Ag triangle structure is $d_{2}$, the thickness of the center of the ITO layer is $l_{2}$.

\section{Theory}

Under the action of an external electromagnetic field, the GaN-Ag periodic grating structure can cause polarized electron oscillation of a specific wavelength [19], and induce the surface plasmon resonance. The dispersion relation is expressed by the following equation:

$$
k \sin \theta \pm n g=\frac{\omega}{c} \sqrt{\frac{\varepsilon_{1} \varepsilon_{2}}{\varepsilon_{1}+\varepsilon_{2}}}=k_{\mathrm{sp}}
$$

where $k \sin \theta$ is the horizontal wave vector of the incident light, $g=2 \pi / T$ is the grating Bragg vector, $T$ is the grating period, $k_{\mathrm{sp}}$ is the wave vector of SPs, $n$ is the diffraction order, $\varepsilon_{1}$ and $\varepsilon_{2}$ are the permittivity of air and $\mathrm{Ag}$, respectively.

The SPs is induced by the attenuated wave generated inside GaN. Due to the total reflection of light on the upper surface of $\mathrm{GaN}$, a part of attenuated wave will penetrate to the lower surface of the Ag film at the interface between GaN and Ag. When $k_{x}$ is coupled with $k_{\mathrm{sp}}$, the plasmon resonance on the lower surface of the Ag film can be induced $[20,21]$. The relationship is expressed as follows:

$$
k_{x}=\frac{\omega}{c} \sqrt{\varepsilon_{3}} \sin \theta=\frac{\omega}{c} \sqrt{\frac{\varepsilon_{1} \varepsilon_{2}}{\varepsilon_{1}+\varepsilon_{2}}}=k_{\mathrm{sp}}
$$

where $\varepsilon_{3}$ is the permittivity of $\mathrm{GaN}, k_{x}$ is the horizontal wave vector of the attenuated wave.

For traditional LEDs, the relationship of the internal quantum efficiency $\eta_{\text {int }}$ and external quantum efficiency $\eta_{\mathrm{ext}}$ are expressed as

$$
\eta_{\text {int }}=\frac{k_{\text {rad }}}{k_{\text {rad }}+k_{\text {non-rad }}}
$$




$$
\eta_{\mathrm{ext}}=C_{\mathrm{ext}} \eta_{\mathrm{int}}
$$

where $C_{\text {ext }}$ is the light extraction efficiency, and $k_{\text {rad }}$ and $k_{\text {non-rad }}$ are the radiation recombination rate and the non-radiation recombination rate of the electron/hole pairs in QW, respectively.

For surface-plasmon-enhanced GaN-LEDs, the relationship of internal quantum efficiency $\eta_{\text {int }}^{\prime}$ and external quantum efficiency $\eta_{\text {ext }}^{\prime}$ are as follows [22]:

$$
\begin{aligned}
& \eta_{\mathrm{int}}^{\prime}=\frac{k_{\mathrm{rad}}+k_{\mathrm{spp}}}{k_{\mathrm{rad}}+k_{\mathrm{spp}}+k_{\text {non-rad }}}=1-\frac{1-\eta_{\mathrm{int}}}{F_{\mathrm{p}}} \\
& \eta_{\mathrm{ext}}^{\prime}=\frac{C_{\mathrm{ext}} k_{\mathrm{rad}}+C_{\mathrm{spp}} k_{\mathrm{spp}}}{k_{\mathrm{rad}}+k_{\mathrm{spp}}+k_{\mathrm{non}-\mathrm{rad}}}
\end{aligned}
$$

where $k_{\mathrm{spp}}$ represents the coupling rate of the QW and SPs; $C_{\mathrm{spp}}$ represents the extraction efficiency of SPP; the Purcell factor $F_{\mathrm{p}}$ represents the quantification of the internal quantum efficiency enhancement effect. And $F_{\mathrm{p}}=\left(k_{\mathrm{rad}}+k_{\mathrm{spp}}+k_{\text {non-rad }}\right) /\left(k_{\mathrm{rad}}+k_{\text {non-rad }}\right)$. According to Eqs. (5) and (6), when $\eta_{\mathrm{int}}^{\prime}, C_{\mathrm{ext}}$, and $C_{\mathrm{spp}}$ increase simultaneously, the emission efficiency of the LED will be significantly improved.

Coupling rate $k_{\mathrm{spp}}$ is derived from Fermi's golden law:

$$
k_{\mathrm{spp}}=\frac{2 \pi}{\hbar}|\mathbf{d} \cdot E(a)|^{2} \rho(\hbar \omega)
$$

where $\hbar$ represents the reduced Planck constant, $\mathbf{d}$ represents the momentum of the electron-hole pair, $a$ represents the position of the metal and semiconductor interface relative to QWs, $E(a)$ represents the electric field intensity where SPs are generated, and $\rho(\hbar \omega)$ represents the SP state density.

A strong local electric field can be generated around SPs, providing a high $\rho(\hbar \omega)$, which will cause a surge in $k_{\mathrm{spp}}$. At this time, the relationship between $k_{\mathrm{spp}}, k_{\mathrm{rad}}$ and $k_{\text {non-rad }}$ is as follows: $k_{\mathrm{spp}} \gg k_{\mathrm{rad}}+k_{\text {non-rad }}$. The greater the $E(a)$, the greater the $k_{\mathrm{spp}}$, the higher the internal quantum efficiency. Therefore, we can use the appropriate metal materials, metal shapes, metal sizes, metal arrangement spacing, and the spacing between the metal and QWs to improve the internal quantum efficiency of the LED. The novel LED structure designed in this paper uses an Ag triangle structure to excite SPs, and the excited SPs can be strongly coupled with QWs, thereby improving the internal quantum efficiency of the LED.

In this paper, the proposed LED structures are numerically simulated by COMSOLTM -RF Module. The simulation setup diagrams of the comparison structure and the novel structure are shown in Figs. $2 \mathbf{a}$ and $2 \mathbf{b}$, respectively. The parameterized scanning function is used to analyze the SP mode, radiated power (RP), absorbed power (AP) and electric field distribution of the LED structures. A scattering boundary condition is set at the bottom boundary of the p-GaN to simulate incident light of different directions and different wavelengths, and the wavelength of the incident light is denoted by $\lambda$. 

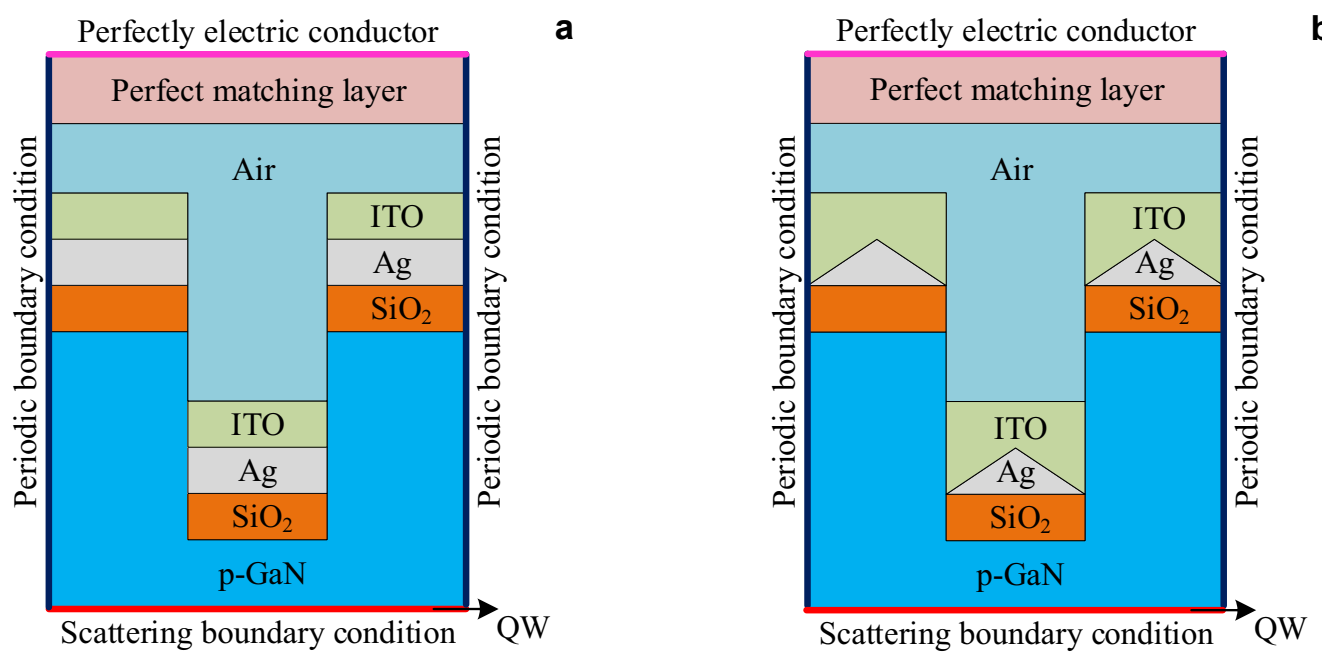

Fig. 2. The simulation setup diagram. (a) The comparison structure, and (b) the novel structure.

A perfect matching layer is provided in the $z$-axis direction to simulate infinitely extending air, and Floquet periodic boundary conditions are set in the $x$ direction and the $-x$ direction. The RP and the AP are obtained by integrating the air layer and the Ag layer, respectively. The $x$-, $y$-, and $z$-axes represent the position coordinates of the LED structure and the color bar represents the magnitude of the electric field intensity. The refractive indices of $\mathrm{GaN}, \mathrm{SiO}_{2}, \mathrm{ITO}$, and air are 2.5, 1.5, 2.0, and 1, respectively, and the refractive indices of $\mathrm{SiO}_{2}$ and ITO are from the data sheet [23]. The permittivity of Ag can be calculated according to

$$
\varepsilon_{m}(\lambda)=\varepsilon_{m r}+i \varepsilon_{m i}=\varepsilon_{\infty}-\frac{\lambda^{2} \lambda_{\mathrm{c}}}{\lambda_{\mathrm{p}}^{2}\left(\lambda_{\mathrm{c}}+i \lambda\right)}
$$

where $\lambda_{\mathrm{p}}$ represents the plasmon wavelength of the metal, $\lambda_{\mathrm{c}}$ represents the resonant wavelength of the metal, and its unit is meter. For Ag: $\varepsilon_{\infty}=5.8, \lambda_{\mathrm{p}}=1.4541 \times 10^{-7} \mathrm{~m}$, $\lambda_{\mathrm{c}}=1.7614 \times 10^{-6} \mathrm{~m}$.

\section{Simulation results and numerical analysis}

For the comparison structure, the influence of the period $\left(T_{2}\right)$, the thickness of $\mathrm{SiO}_{2}\left(h_{1}\right)$ and ITO $\left(l_{1}\right)$ on the emission efficiency are studied. Since the incident light cannot directly induce the SPs, the grating structure can cause polarization electron oscillations of a specific wavelength to provide an additional horizontal wave vector for the excitation of the SPs. Therefore, the grating period has a significant effect on the induced SPs, and the SPs can be effectively induced when the grating period is within a certain range. At 
the same time, the grating period can also affect the escape angle of $\mathrm{GaN}$ and improve the light extraction efficiency. First, the RP and AP at different periods of $T_{1}$ are shown in Fig. $3 \mathbf{a}$ for the case of $h_{1}=20 \mathrm{~nm}, d_{1}=20 \mathrm{~nm}, l_{1}=20 \mathrm{~nm}, \lambda=460 \mathrm{~nm}$. The subgraph in Fig. 3a can clearly show the peak value of $T_{1}$ in the range of $140-160 \mathrm{~nm}$, and the $\mathrm{RP}$ and AP reach the highest peak at the same time when $T_{1}=149 \mathrm{~nm}$. The RP and AP at different thickness of $h_{1}$ are shown in Fig. $3 \mathbf{b}$ for the case of $T_{1}=149 \mathrm{~nm}, d_{1}=20 \mathrm{~nm}$, $l_{1}=20 \mathrm{~nm}, \lambda=460 \mathrm{~nm}$, and the RP and AP reach the highest peak at the same time when $h_{1}=20 \mathrm{~nm}$. The RP and AP at different thickness of $l_{1}$ are shown in Fig. $3 \mathbf{c}$ for the case of $T_{1}=149 \mathrm{~nm}, h_{1}=20 \mathrm{~nm}, d_{1}=20 \mathrm{~nm}, \lambda=460 \mathrm{~nm}$, and the RP and AP reach the highest peak at the same time when $l_{1}=20 \mathrm{~nm}$. The RP and AP at different wavelengths of $\lambda$ are shown in Fig. $3 \mathbf{d}$ for the case of $T_{1}=149 \mathrm{~nm}, h_{1}=20 \mathrm{~nm}, d_{1}=20 \mathrm{~nm}$,
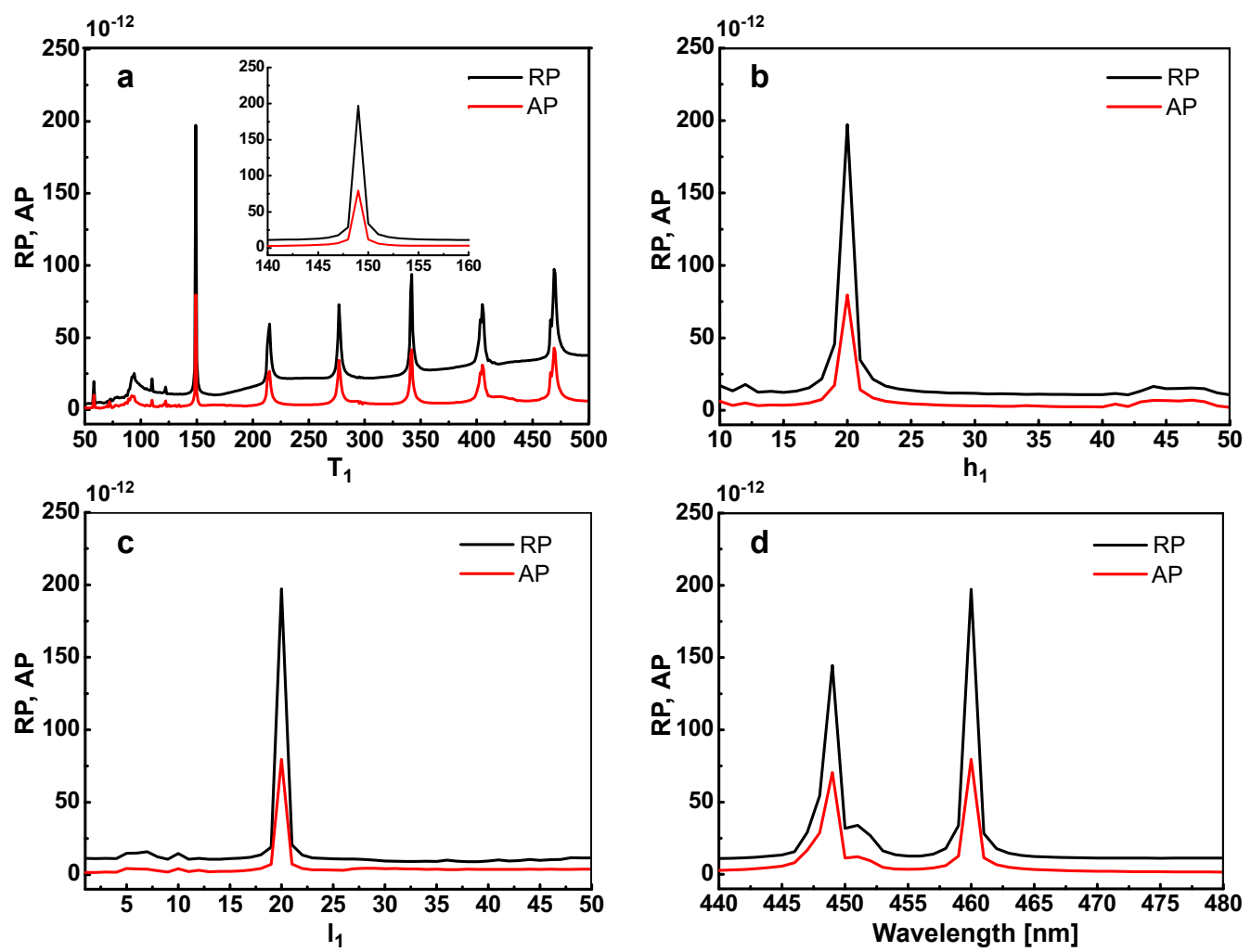

Fig. 3. (a) The RP and AP at different period $T_{1}$. (b) The RP and AP at different thickness of $h_{1}$. (c) The RP and AP at different thickness of $l_{1}$. (d) The RP and AP at different wavelength. (e) The distribution of the electric field at the wavelength of $449 \mathrm{~nm}$. (f) The distribution of the electric field at the wavelength of $460 \mathrm{~nm}$. (a) $h_{1}=20 \mathrm{~nm}, d_{1}=20 \mathrm{~nm}, l_{1}=20 \mathrm{~nm}, \lambda=460 \mathrm{~nm}$, (b) $T_{1}=149 \mathrm{~nm}, d_{1}=20 \mathrm{~nm}, l_{1}=20 \mathrm{~nm}$, $\lambda=460 \mathrm{~nm}$, (c) $T_{1}=149 \mathrm{~nm}, h_{1}=20 \mathrm{~nm}, d_{1}=20 \mathrm{~nm}, \lambda=460 \mathrm{~nm}$, (d) $T_{1}=149 \mathrm{~nm}, h_{1}=20 \mathrm{~nm}, d_{1}=$ $=20 \mathrm{~nm}, l_{1}=20 \mathrm{~nm},(\mathbf{e}) T_{1}=149 \mathrm{~nm}, h_{1}=20 \mathrm{~nm}, d_{1}=20 \mathrm{~nm}, l_{1}=20 \mathrm{~nm}, \lambda=449 \mathrm{~nm}$, and (f) $T_{1}=$ $=149 \mathrm{~nm}, h_{1}=20 \mathrm{~nm}, d_{1}=20 \mathrm{~nm}, l_{1}=20 \mathrm{~nm}, \lambda=460 \mathrm{~nm}$. 

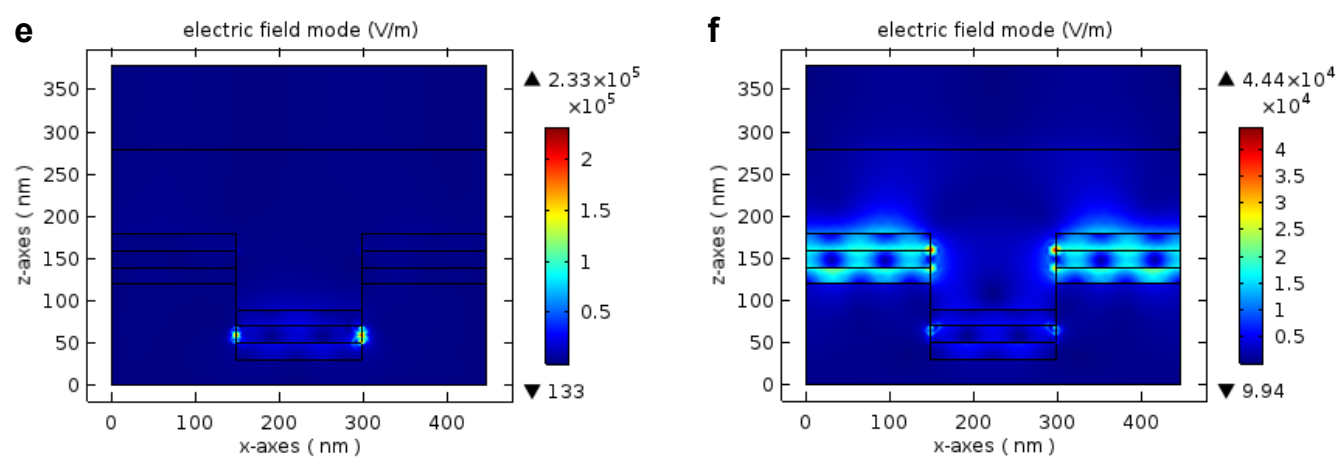

Fig. 3. Continued.

$l_{1}=20 \mathrm{~nm}$, and the RP and AP reach the highest peak at the same time when $\lambda=460 \mathrm{~nm}$. When $T_{1}=149 \mathrm{~nm}, h_{1}=20 \mathrm{~nm}, d_{1}=20 \mathrm{~nm}, l_{1}=20 \mathrm{~nm}, \lambda=460 \mathrm{~nm}$, the RP and AP reach the highest peak, the SPs and QW are strongest coupled and the light extraction efficiency reaches the maximum. The distribution of the electric field at the wavelength of 449 and $460 \mathrm{~nm}$ are shown in Fig. 3e and 3f, respectively.

For the novel structure, the influence of the period $\left(T_{2}\right)$, the thickness of $\mathrm{SiO}_{2}\left(h_{2}\right)$, the height of the Ag triangle structure $\left(d_{2}\right)$ and the thickness of the center of the ITO layer $\left(l_{2}\right)$ on the emission efficiency are studied. First, the RP and AP at different periods of $T_{2}$ are shown in Fig. $4 \mathbf{a}$ for the case of $h_{2}=17 \mathrm{~nm}, d_{2}=20 \mathrm{~nm}, l_{2}=10 \mathrm{~nm}$, $\lambda=460 \mathrm{~nm}$. The subgraph in Fig. 3a can clearly show the peak value of $T_{2}$ in the range of $150-165 \mathrm{~nm}$, and the RP and AP reach the highest peak at the same time when $T_{2}=156 \mathrm{~nm}$. The RP and AP at different thicknesses of $h_{2}$ are shown in Fig. $4 \mathbf{b}$ for the case of $T_{2}=156 \mathrm{~nm}, d_{2}=20 \mathrm{~nm}, l_{2}=10 \mathrm{~nm}, \lambda=460 \mathrm{~nm}$, and the RP and AP reach the highest peak at the same time when $h_{2}=17 \mathrm{~nm}$. The RP and AP at different heights of $d_{2}$ are shown in Fig. $4 \mathbf{c}$ for the case of $T_{2}=156 \mathrm{~nm}, h_{2}=17 \mathrm{~nm}, l_{2}=10 \mathrm{~nm}, \lambda=$ $=460 \mathrm{~nm}$, and the RP and AP reach the highest peak at the same time when $d_{2}=20 \mathrm{~nm}$. The RP and AP at different thicknesses of $l_{2}$ are shown in Fig. $4 \mathbf{d}$ for the case of $T_{2}=$ $=156 \mathrm{~nm}, h_{2}=17 \mathrm{~nm}, d_{2}=20 \mathrm{~nm}, \lambda=460 \mathrm{~nm}$, and the RP and AP reach the highest peak at the same time when $l_{2}=10 \mathrm{~nm}$. The RP and AP at different wavelength of $\lambda$ are shown in Fig. $4 \mathbf{e}$ for the case of $T_{2}=156 \mathrm{~nm}, h_{2}=17 \mathrm{~nm}, d_{2}=20 \mathrm{~nm}, l_{2}=10 \mathrm{~nm}$, and the RP and AP reach the highest peak at the same time when $\lambda=460 \mathrm{~nm}$. When $T_{2}=156 \mathrm{~nm}, h_{2}=17 \mathrm{~nm}, d_{2}=20 \mathrm{~nm}, l_{2}=10 \mathrm{~nm}, \lambda=460 \mathrm{~nm}$, the RP and AP reach the highest peak, the SPs and QW are strongest coupled and the light extraction efficiency reaches the maximum, and the emission efficiency is nearly 4 times that of the comparison structure. The distribution of the electric field at the wavelength of $460 \mathrm{~nm}$ is shown in Fig. 4f. Compared with the comparison structure, the Ag triangle structure introduced by the novel structure can further enhance the coupling between SPs and QWs, and can also improve the light extraction efficiency and the emission efficiency reaches the maximum. 

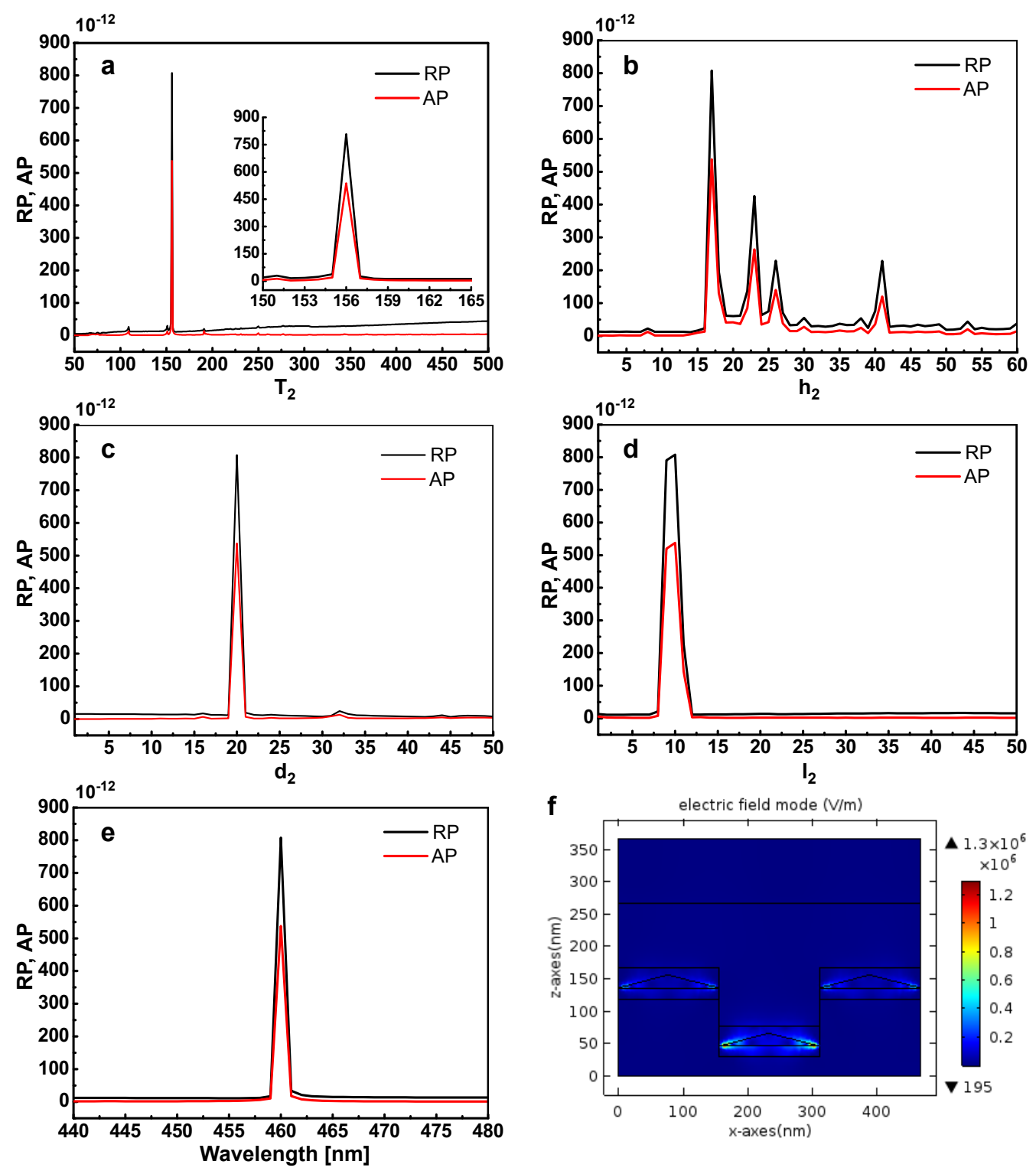

Fig. 4. (a) The RP and AP at different period $T_{2}$. (b) The RP and AP at different thickness of $h_{2}$. (c) The RP and AP at different height of $d_{2}$. (d) The RP and AP at different thickness of $l_{2}$. (e) The RP and AP at different wavelength. (f) The distribution of the electric field at the wavelength of $460 \mathrm{~nm}$. (a) $h_{2}=17 \mathrm{~nm}$, $d_{2}=20 \mathrm{~nm}, l_{2}=10 \mathrm{~nm}, \lambda=460 \mathrm{~nm}$, (b) $T_{2}=156 \mathrm{~nm}, d_{2}=20 \mathrm{~nm}, l_{2}=10 \mathrm{~nm}, \lambda=460 \mathrm{~nm}$, (c) $T_{2}=156 \mathrm{~nm}$, $h_{2}=17 \mathrm{~nm}, l_{2}=10 \mathrm{~nm}, \lambda=460 \mathrm{~nm},(\mathbf{d}) T_{2}=156 \mathrm{~nm}, h_{2}=17 \mathrm{~nm}, d_{2}=20 \mathrm{~nm}, \lambda=460 \mathrm{~nm},(\mathbf{e}) T_{2}=156 \mathrm{~nm}$, $h_{2}=17 \mathrm{~nm}, d_{2}=20 \mathrm{~nm}, l_{2}=10 \mathrm{~nm}$, (f) $T_{2}=156 \mathrm{~nm}, h_{2}=17 \mathrm{~nm}, d_{2}=20 \mathrm{~nm}, l_{2}=10 \mathrm{~nm}, \lambda=460 \mathrm{~nm}$.

The work [24] proposed a high emission efficiency SPs-enhanced GaN-based LED, in which an ITO layer is deposited on a trapezoidal $\mathrm{p}-\mathrm{GaN}$ layer and a periodic Ag grating is fabricated on the top of the ITO layer. Compared with the work [24], the $\mathrm{SiO}_{2}$ layer 
and ITO layer introduced in the novel LED designed in this paper can form a quasi-symmetrical waveguide structure, which further improves the internal quantum efficiency and light extraction efficiency of the LED. In addition, the metal Ag at the bottom of the p-GaN groove in the novel LED is closer to QWs, the coupling between SPs and QWs is stronger, and the internal quantum efficiency is higher. Therefore, the novel LED in this paper will have more advantages in improving the emission efficiency of LEDs.

\section{Conclusions}

In this paper, the novel structure mainly contains a $\mathrm{SiO}_{2}$ film, an Ag layer and an ITO layer coated on the rectangularly-patterned $\mathrm{p}-\mathrm{GaN}$ layer sequentially. The Ag layer can induce SPs and enhance the internal quantum efficiency. The $\mathrm{SiO}_{2}$ film can suppress the absorption loss of SPs, and further improve the light extraction efficiency and the SPs extraction efficiency. The ITO layer can be used as a buffer layer of LED for its high light transmittance and conductivity. The $\mathrm{SiO}_{2}$ film, Ag layer and ITO layer can form a quasi-symmetric waveguide structure, which can extend the near-field distribution range of the local electric field, reduce the loss and absorption of the metal to the SPs, and improve the extraction efficiency of the SPs. At the same time, we designed a comparison structure for comparative analysis. The COMSOL software is used to simulate and calculate these LED structures based on the finite element method. The radiated powers, the absorbed powers and the distribution of the electric field are obtained and analyzed. The results reveal that the emission efficiency of the novel GaN-LED can be increased to nearly 4 times compared with the comparison structure.

Acknowledgment - This work was supported by the Local Science and Technology Development Fund Projects Guided by the Central Government, China (No. 206Z1703G), the Scientific Research Project of Colleges and Universities in Hebei Province, China (No. QN2019061), and the Fundamental Research Funds for Provincial Universities, China (No. 2021JK03).

\section{References}

[1] Li Z., Xue D., Liu D., Liu Y., Ye W., Zhang Y., Wang Y., Zheng C., Portable visible light communication transmitter and receiver using core-shell CdSe/ZnS quantum dots white light-emitting diode, IET Communications 13(7), 2019, pp. 873-878, DOI: 10.1049/iet-com.2018.5303.

[2] Hea J., Wu K., He J., Zhou Z., Ma J., Shi J., An efficient encoder-subcarrier mapping method combined with polar code for visible light communication, IEEE Access 7, 2019, pp. 69119-69125, DOI: 10.1109/ACCESS.2019.2916005.

[3] Xu J., Zhang W., Peng M., DAI J., Chen C., Light-extraction enhancement of GaN-based $395 \mathrm{~nm}$ flip-chip light-emitting diodes by an Al-doped ITO transparent conductive electrode, Optics Letters 43(11), 2018, pp. 2684-2687, DOI: 10.1364/OL.43.002684.

[4] PARK J., LeE J.H., Bending effect on the circular polarizer of an organic light-emitting diode display, Applied Optics 58(13), 2019, pp. 3671-3675, DOI: 10.1364/AO.58.003671.

[5] Okamoto K., Niki I., Shvartser A., Narukawa Y., Mukai T., Scherer A., Surface-plasmon-enhanced light emitters based on InGaN quantum wells, Nature Materials 3(9), 2004, pp. 601-605, DOI: 10.1038/nmat1198. 
[6] Окamoto K., Niki I., Scherer A., Narukawa Y., Mukai T., Kawakami Y., Surface plasmon enhanced spontaneous emission rate of In GaN/GaN quantum wells probed by time-resolved photoluminescence spectroscopy, Applied Physics Letters 87(7), 2005, article 071102, DOI: 10.1063/ 1.2010602 .

[7] Okamoto K., Niki I., Shvartser A., Maltezos G., Narukama Y., Mukai T., Kawakami Y., Scherer A., Surface plasmon enhanced bright light emission from InGaN/GaN, Physica Status Solidi 204(6), 2007, pp. 2103-2017, DOI: 10.1002/pssa.200674856.

[8] Yeh D.M., Huang C.F., Lu Y.C., Chen C.Y., Tang T.-Y., Huang J.-J., Shen K.-C., Yang Y.-J., YANG C.C., Surface plasmon leakage in its coupling with an InGaN/GaN quantum well through an ohmic contact, Applied Physics Letters 91(6), 2007, article 063121, DOI: 10.1063/1.2768913.

[9] Yen D.M., Huang C.F., Chen C.Y., Lu Y.C., YANG C.C., Localized surface plasmon-induced emission enhancement of a green light-emitting diode, Nanotechnology 19(34), 2008, article 345201, DOI: $10.1088 / 0957-4484 / 19 / 34 / 345201$.

[10] Cho C.Y., Kwon M.K., Lee S.J., Han S.H., Kang J.W., Kang S.E., Lee D.Y., Park S.J., Surface plasmon-enhanced light-emitting diodes using silver nanoparticles embedded in $p$-GaN, Nanotechnology 21(20), 2010, article 205201, DOI: 10.1088/0957-4484/21/20/205201.

[11] Cho C.Y., Kim K.S., Lee S.J., Kwon M.K., Ko H., Kim S.T., Jung G.Y., Park S.J., Surface plasmon -enhanced light-emitting diodes with silver nanoparticles and $\mathrm{SiO}_{2}$ nano-disks embedded in $\mathrm{p}$-GaN, Applied Physics Letters 99(4), 2011, article 041107, DOI: 10.1063/1.3616149.

[12] Shen K.C., Liao C.H., Yu Z.Y., Wang J.Y., Lin C.H., Kiang Y.W., Yang C.C., Effects of the intermediate $\mathrm{SiO}_{2}$ layer on polarized output of a light-emitting diode with surface plasmon coupling, Journal of Applied Physics 108(11), 2010, article 113101, DOI: 10.1063/1.3517082.

[13] KaO C.C., Su Y.K., Lin C.L., CHEN J.J., Localized surface plasmon-enhanced nitride-based light-emitting diode with Ag nanotriangle array by nanosphere lithography, IEEE Photonics Technology Letters 22(13), 2010, pp. 984-986, DOI: 10.1109/LPT.2010.2049013.

[14] Zhang H., Zhu J., Zhu Z., Li Q., JiN G., Surface-plasmon-enhanced GaN-LED based on the quasi-symmetrical planar waveguide structure, Optics Communications 311, 2013, pp. 311-316, DOI: 10.1016/ j.optcom.2013.08.078.

[15] Zhang H., Zhu J., Zhu Z., Jin Y., Li Q., Jin G., Surface-plasmon-enhanced GaN-LED based on a multilayered M-shaped nano-grating, Optics Express 21(11), 2013, pp. 13492-13501, DOI: 10.1364/ OE.21.013492.

[16] Zhu J., Zhang H., Zhu Z., Li Q., Jin G., Surface-plasmon-enhanced GaN-LED based on the multilayered rectangular nano-grating, Optics Communications 322, 2014, pp. 66-72, DOI: $10.1016 /$ j.optcom.2014.02.011.

[17] Yao Y.F., Lin C.H., Chao C.Y., Chang W.Y., Su C.Y., Tu C.G., Kiang Y.W., Yang C.C., Coupling of a light-emitting diode with surface plasmon polariton or localized surface plasmon induced on surface silver gratings of different geometries, Optics Express 26(7), 2018, pp. 9205-9219, DOI: 10.1364/OE.26.009205.

[18] Hong S.H., Kim N.Y., Kang J.W., Kim J.J., Jung Y.S., Kim D.Y., Yim S.Y., Park S.J., Quantum efficiency enhancement depending on the thickness of p-GaN spacer layer in localized surface plasmon-enhanced near-ultraviolet light-emitting diodes by using colloidal silver nanoparticles, ECS Journal of Solid State Science and Technology 9(1), 2020, article 016003, DOI: 10.1149 / 2.0042001 JSS.

[19] Lin T.H., Wang S.J., Tu Y.C., Hung C.H., Yu T.H., Improving the performance of power GaN-based thin-film flip-chip LEDs through a twofold roughened surface, Materials Science in Semiconductor Processing 45, 2016, pp. 69-75, DOI: 10.1016/j.mssp.2016.01.010.

[20] Отто A., Excitation of nonradiative surface plasma waves in silver by the method of frustrated total reflection, Zeitschrift für Physik A Hadrons and nuclei 216(4), 1968, pp. 398-410, DOI: $10.1007 /$ BF01391532. 
[21] Li W.C., Sha X.P., Li Z.Q., Meng X.Y., Gu E., Emission enhancement of light-emitting diode by localized surface plasmon induced by Ag inserts in p-GaN and $\mathrm{TiO}_{2}-\mathrm{Ag}$ grating, Plasmonics 12(6), 2017, pp. 1855-1860, DOI: 10.1007/s11468-016-0454-4.

[22] Li Z., XIE R., Li X., Gu E., Niu L., SHA X., Luminous enhancement of nitride light-emitting diodes by localized surface plasmon and triangular structure, Superlattices and Microstructures 120, 2018, pp. 127-135, DOI: 10.1016/j.spmi.2018.05.031.

[23] Palik E.D., Handbook of Optical Constants of Solids, Academic Press, New York, 1998, pp. 52-63.

[24] Guo S.L., Li X., Li Z.Q., Gu E., Zнао X.T., Improving the luminous efficiency of gallium nitride -based light-emitting diodes using Ag nanograting structure, Journal of Nanophotonics 13(4), 2019, article 46010, DOI: $10.1117 / 1 . J N P .13 .046010$.

Received August 11, 2020

in revised form December 14, 2020 